\title{
Effect of Endurance Exercise with Garlic Supplement Consumption on Intracellular and Vascular Adhesion Molecules in Sedentary Women
}

\section{ARTICLE INFO}

\section{Article Type}

Original Research

\section{Authors}

Soori R.* $P h D$,

Choopani Z. ${ }^{1} M S c$

Falahian N. ${ }^{2} M S C$,

Choopani S. ${ }^{3} M S C$,

Ramezankhani A. ${ }^{3} \mathrm{PhD}$

\begin{tabular}{l} 
How to cite this article \\
\hline Soori R, Choopani Z, Falahian N, \\
Choopani S, Ramezankhani A. \\
Effect of Endurance Exercise \\
with Garlic Supplement \\
Consumption on Intracellular \\
and Vascular Adhesion \\
Molecules in Sedentary Women. \\
Horizon of Medical Sciences. \\
2017;23(1):85-91.
\end{tabular}

*Sport Physiology Department, Education \& Sport Sciences Faculty, Tehran University, Tehran, Iran ${ }^{1}$ Sport Physiology Department, Education \& Sport Sciences Faculty, Science \& Research Branch, Islamic Azad University, Tehran, Iran ${ }^{2}$ Sport Physiology Department, Education \& Sport Sciences Faculty, Alzahra University, Tehran, Iran ${ }^{3}$ Sport Physiology Department, Education \& Sport Sciences Faculty, Tehran University, Tehran, Iran

\section{Correspondence}

Address: Faculty of Physical Education and Sport Sciences, Tehran University, between 15thand 16thStreet, North Kargar Street, Tehran, Iran

Phone: +98 (21) 61118859

Fax: +98 (21) 88351741

soori@ut.ac.ir

\section{Article History}

Received: April 20, 2016

Accepted: July 19, 2016

ePublished: January 19, 2017

\section{A B S T R A C T}

Aims The intercellular adhesion molecule 1 (ICAM-1) and the vascular cell adhesion molecule 1 (VCAM-1) play important roles in the pathogenesis of atherosclerosis. The aim of the study was to investigate the effects of the sport activities with garlic supplementation on the levels of ICAMs and VCAMs in the sedentary women.

Materials \& Methods In the pretest-posttest semi-experimental study, 40 over-weight women referred to the health clinics in western Tehran were studied in 2015. The subjects, selected via random sample selecting method, were randomly divided into four 10-person groups including sport exercise, exercise with garlic supplementation, supplementation, and control. Two 500mg supplementation capsules were daily administrated. In addition, including 5 sessions a week, 10 -week $60-75 \%$ of maximum heart beat aerobic activity was conducted. The anthropometric indices, the levels of the adhesion molecules, and blood lipids of the subjects were measured at the beginning and 48 hours after the end of the exercises. Data was analyzed by SPSS 16 software using two-way ANOVA, Tukey's post-hoc, and dependent $\mathrm{T}$ tests.

Findings The ICAM-1 levels in exercise + supplementation and exercise groups, and the VCAM-1 levels in exercise + supplementation and supplementation groups were significantly reduced at the posttest stage compared to the pretest stage, as well as to control group. In addition, the mean weight, lipid percentage, BMI, and LDL-C in exercise and exercise + supplementation groups were significantly reduced. Nevertheless, the cholesterol level was significantly reduced in exercise + supplementation group only $(\mathrm{p}<0.05)$.

Conclusion 10-week sport activity with garlic supplementation reduces the levels of ICAM and VCAM in the sedentary women.

Keywords Vascular Cell Adhesion Molecule-1; Intercellular Adhesion Molecule-1; Garlic Women

\section{T A T I O N L I N KS}

[1] The effects of three months aerobic exercise on ... [2] The effects of 8-week aerobic exercises on ... [3] Overview of epidemiology and contribution of ... [4] Effects of exercise training on ... [5] Inflammatory markers and cardiovascular health in older adults ... [6] Exercise in the metabolic... [7] Exercise preconditioning ameliorates inflammatory injury in ischemic rats during... [8] Moderate regular exercise increases basal production of nitric oxide in elderly ... [9] Effect of upper-lower- limb exercise training on circulation soluble adhertion molecules, hs-CRP and stress protein in pasint with ... [10] Effect of an exercise program, on hemodynamic, metabolic and inflammatory markers in obese Mexican ... [11] The effect of physical activity or exercise on key biomarkers in atherosclerosis--a systematic ... [12] Effect of twelve weeks aerobic training on selected molecules TNF$1 \alpha$, CRP, ICAM- 1 and VCAM-1 in type 2 diabetes in middle-aged ... [13] Femoral artery ... [14] Preventive effects of ... [15] Aged garlic extract ameliorates physical ... [16] The effect of endurance training and garlic consumption on vo2max in non-active ... [17] Antioxidant effect of garlic and aged black garlic in ... [18] Effects of garlic consumption on physiological variables and ... [19] The effect of garlic supplementation on aerobic performance in non-athlete ... [20] Garlic and onion attenuates vascular inflammation and oxidative stress in ... [21] Anti-wrinkle and anti-inflammatory effects of active garlic components and ... [22] Chloroform extract of aged black garlic attenuates TNF- $\alpha$-induced ROS ... [23] Immunomodulation and anti-inflammatory effects of ... [24] Effect of exercise training on ... [25] Effect of Aerobic Training with Omega-3 consumption on ... [26] Effect of sprint training and detraining period on ... [27] Effects of progressive and maximal ... [28] Simple advice on lifestyle ... [29] Effect of garlic powder on C-reactive protein and ... [30] Effect of garlic powder on C-reactive protein and plasma lipids in overweight and smoking ... 
فعالشدن اندوتليال و سنتز يروتئينهاى ييشالتهابى مانند

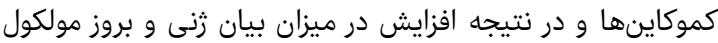

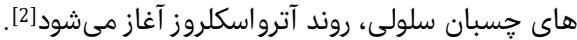

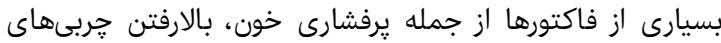

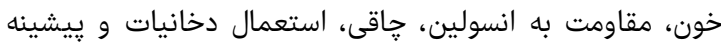

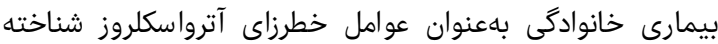

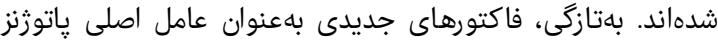

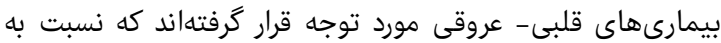

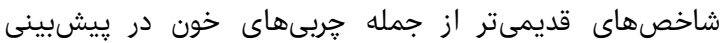

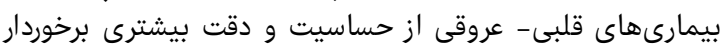

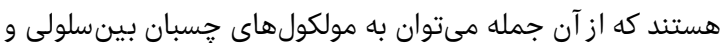

عروقى اشاره نمود [3]. ICAM-1

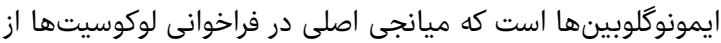

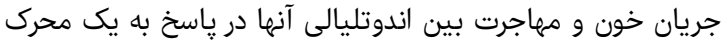

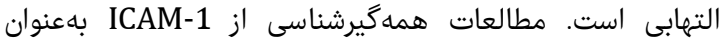

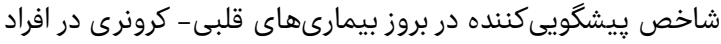

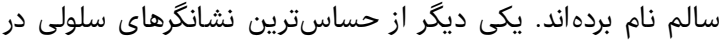

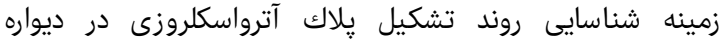

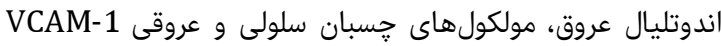

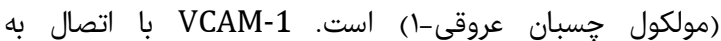
مونوسيتها و حركت آنها به عمقا) اله اندوتليال، روند تشكيل

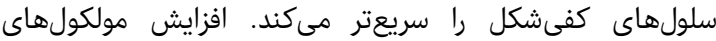

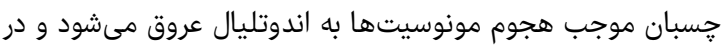

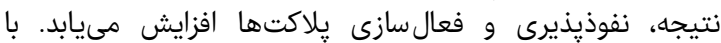

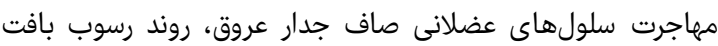

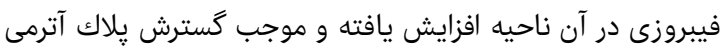

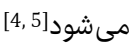

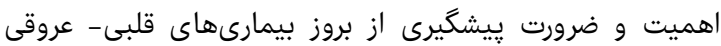

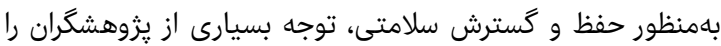

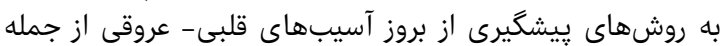

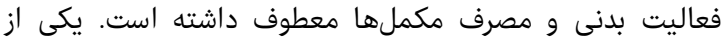

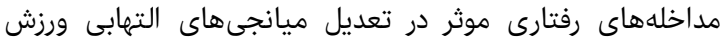

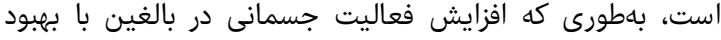

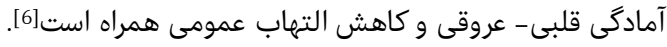

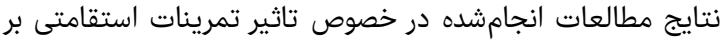

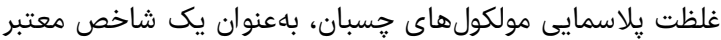

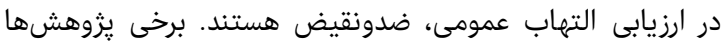

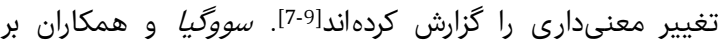

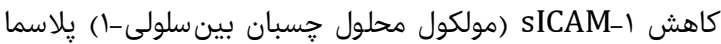

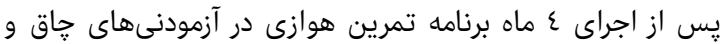

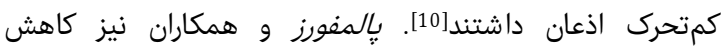
معنى دارى را در سطح مولكولهاى جسبان إنسان

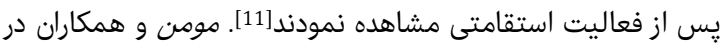

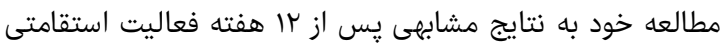

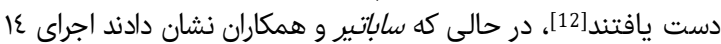

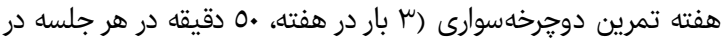

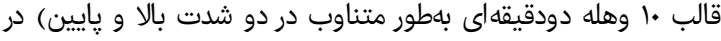
زنان سالم و غيرفعال تغيير معنىدارى در سطح

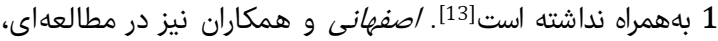

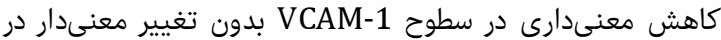
سطوح ICAM-1 سرم يֶ از فعاليت هوازى را نشان دادند [14]. در

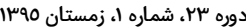

تاثير تمرين استقامتى همراه با مصرف مكمل سير

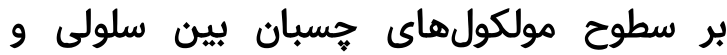
عروقى در زنان كمتحرك مولى

PhD " رحمان سورى

كروه فيزيولوزى ورزش، دانشكده تربيت بدنى و علوم ورزشى، دانشكاه تهران،

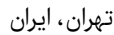

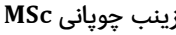

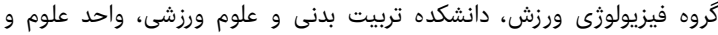
تحقيقات، دانشگاه آزاد اسلامى، تهران ، ايران

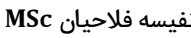

كروه فيزيولوزى ورزش، دانشكده تركيت فربيت بدنى و علوم ورزشى، دانشكاه الزهرا،

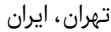

MSc ثروت جويانى ايران

تروه فيزيولوزى ورزش، دانشكده تربيت بدنى و علوم ورزشى، دانشكاه تهران، تهران، ايران

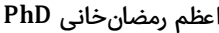

كروه فيزيولوزى ورزش، دانشكان تراني تربيت بدنى و علوم ورزشى، دانشكاه تهران،

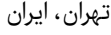

يكيده

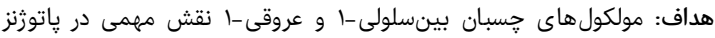

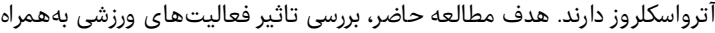

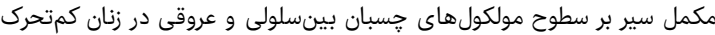

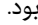

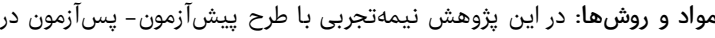

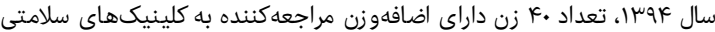

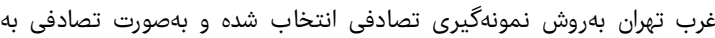

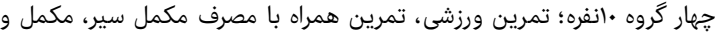

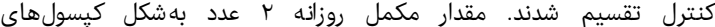

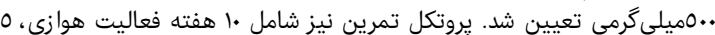

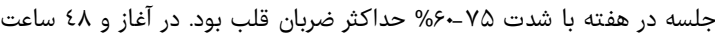

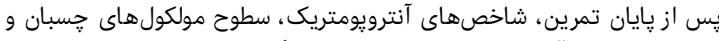

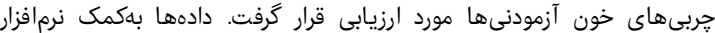

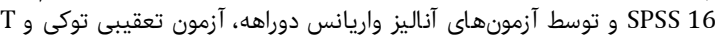

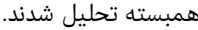

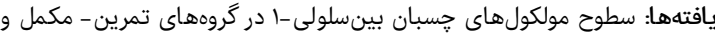

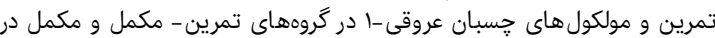

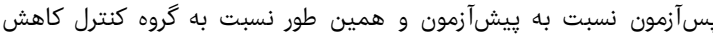

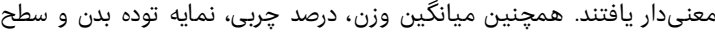
LDL-C

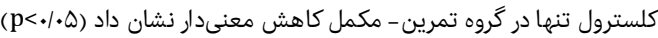

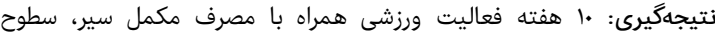

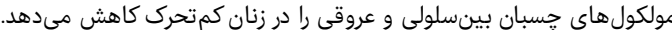

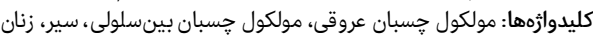

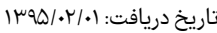

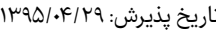
soori@ut.ac.ir :نويسنده مسئول:

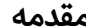

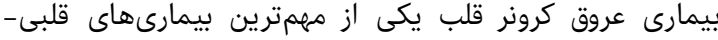

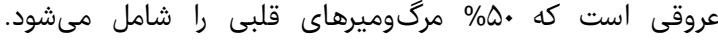

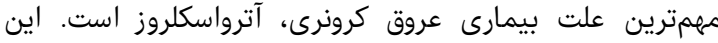

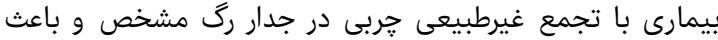

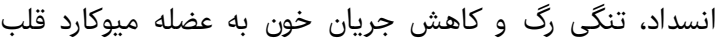

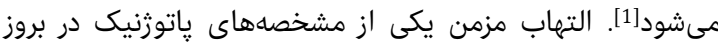
آترواسكلروز است؛ بدين ترتيب كه با آغاز التهاب عروقي باتي و متعاقباً

فصل نامه علمى - يزوهشى افق دانش 


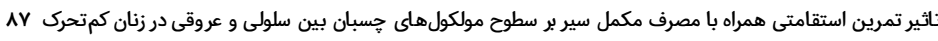

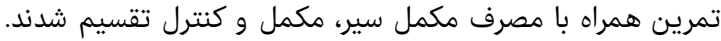

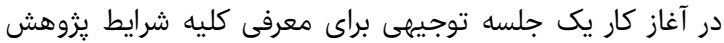

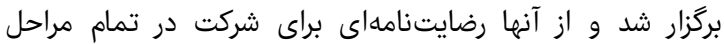

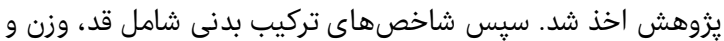

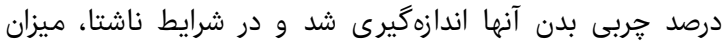

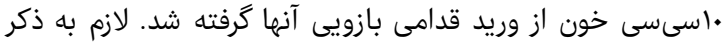

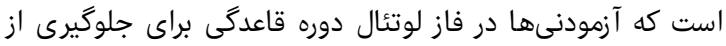

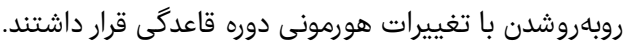

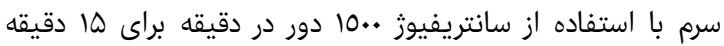

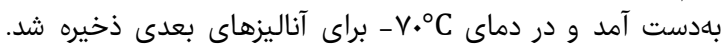

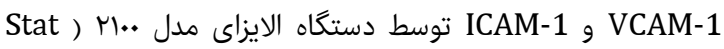
Fax كيتهاى الايزا (شركت BMS232 و BMS232TEN

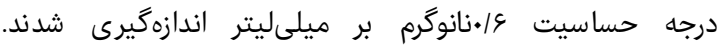

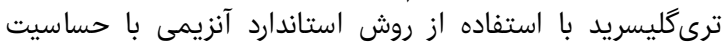

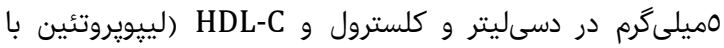

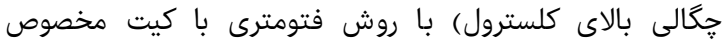

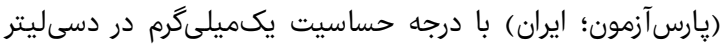

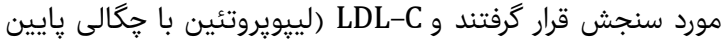

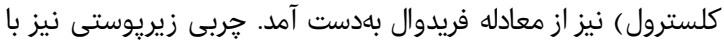

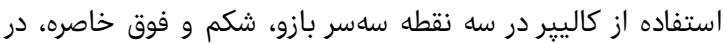

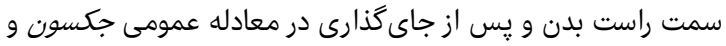

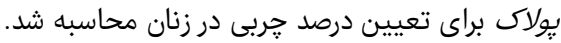

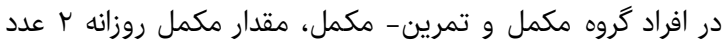

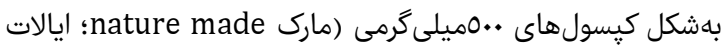

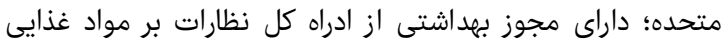

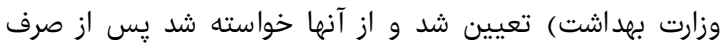

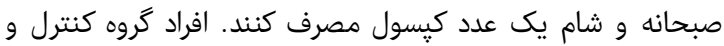

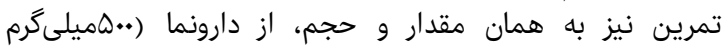

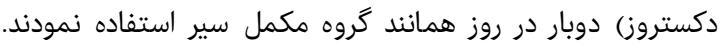

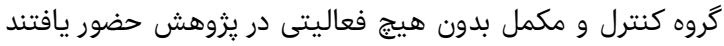

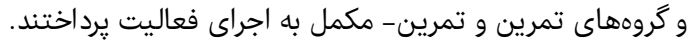

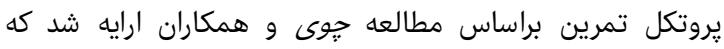

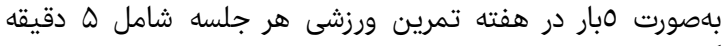

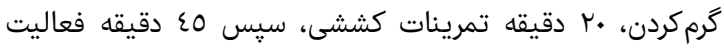

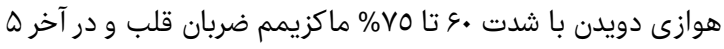

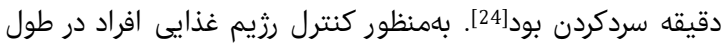

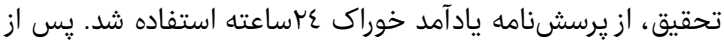

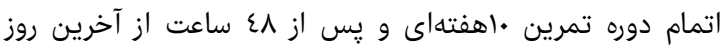

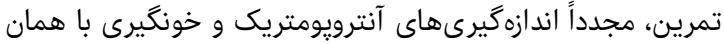

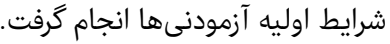

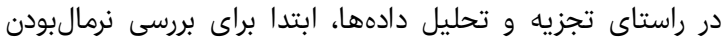

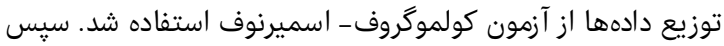

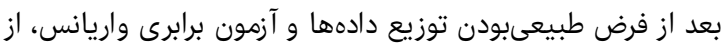

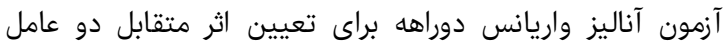

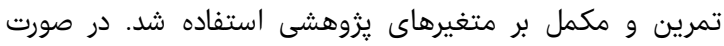

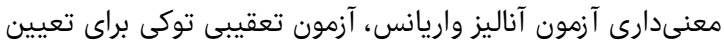

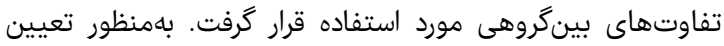

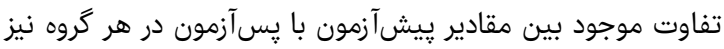

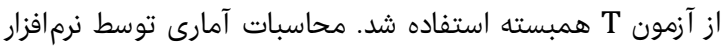

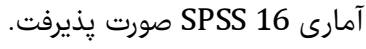

حالى كه نتايج مطالعه حجازى و همكاران، حاكى از كاهش

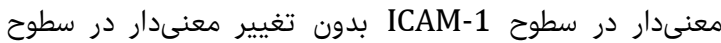
\% سرم يس از 1 هفته فعاليت هوازى با شدت . V.-VAM-1

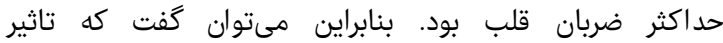

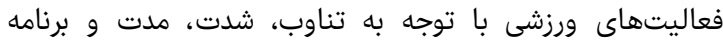

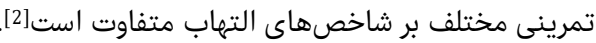

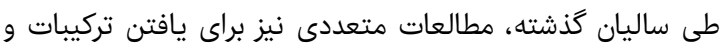

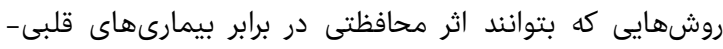

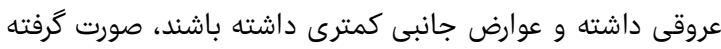

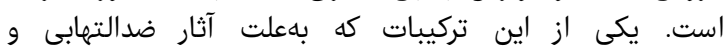

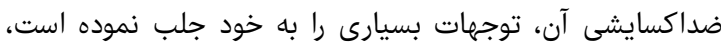

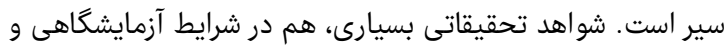

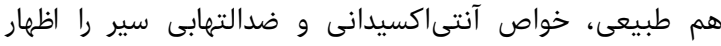

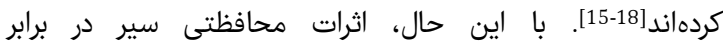

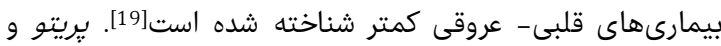

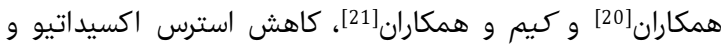
سطوح VCAM-1 را با دريافت عصاره سير نشان دادند. در مطالعه

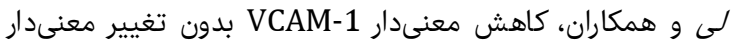

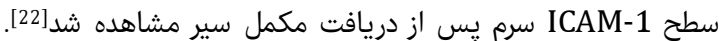

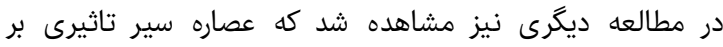

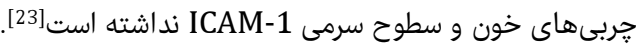

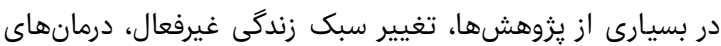

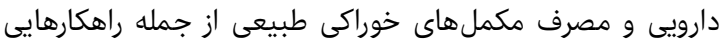

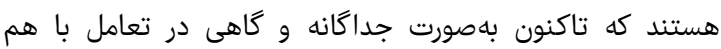

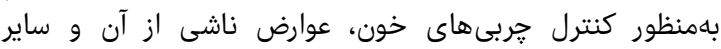

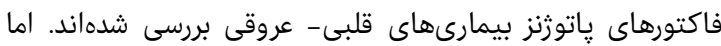

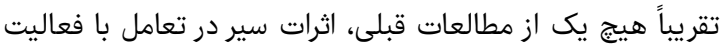
بدنى بر سطح مولكولهاى جسبان ICAM-1 و

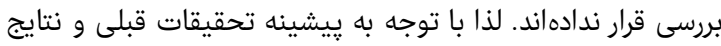

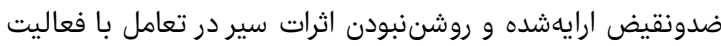

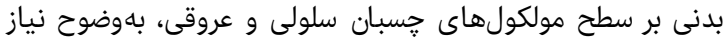

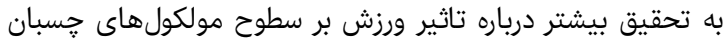
فCAM-1

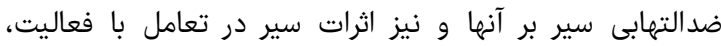
احساس مى شىود. لذا مطالعه حاضر با هدف بررسى تاثير فعاليتهاى ورزشى همراه با

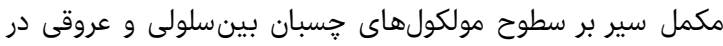
زنان كمتحرك انجام كرفت.

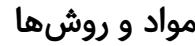

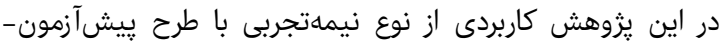

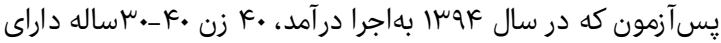

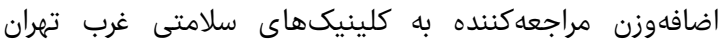
بهروش نمونهگيرى تصادفى انتخاب شدند. شرايط ورود به به مطالعه

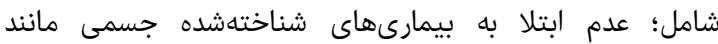

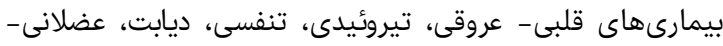

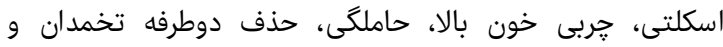

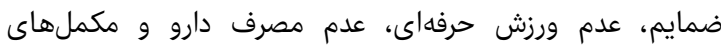

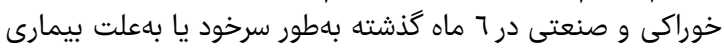

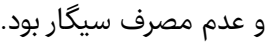
اين افراد بهطور تصادفى به جهار گروه •انفره؛ تمرين ورزشى، 
جد 1 رحمان سورى و همكاران ميانين

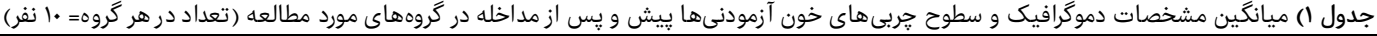

\begin{tabular}{|c|c|c|c|c|}
\hline كروه تمرين - مكمل & كروه تمرين & كروه مكمل & كروه كنترل & متغيرها \\
\hline$\mu\urcorner / r \cdot \pm 7 / r$ & $\mu \varepsilon / \Lambda \cdot \pm V / V$. & $\mu \mu / \Lambda \cdot \pm \varepsilon / \gamma$. & $\mu \varepsilon / \varepsilon \cdot \pm 0 / \varepsilon$. & سن (سال) \\
\hline $\begin{array}{l}70 / r \cdot \pm V / \varepsilon . \\
*_{\top} \tau / r \cdot \pm T / 1 .\end{array}$ & $\begin{array}{l}\mathrm{TV} / 1 \cdot \pm V / 7 \\
*_{\urcorner \varepsilon / V \cdot \pm V / I}\end{array}$ & $\begin{array}{l}70 / \mu \cdot \pm 0 / r . \\
7 \varepsilon / \mu \cdot \pm 0 / r\end{array}$ & $\begin{array}{l}77 / V \cdot \pm V / \varepsilon . \\
77 / r \cdot \pm 7 / 9 .\end{array}$ & 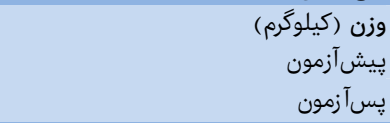 \\
\hline $10 \mathrm{~V} / \cdot . \pm 0 / \mathrm{V}$ & $10 \wedge / 1 \cdot \pm \varepsilon / 1$. & $10 V / r \cdot \pm T / \mu$ & $107 / \varepsilon \cdot \pm 7 / 1$. & قد (سانتىمتر) \\
\hline 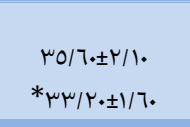 & $\begin{array}{l}\mu\urcorner / \varepsilon \cdot \pm 1 / \varepsilon . \\
*_{\mu \varepsilon / \varepsilon \cdot \pm \cdot / \Lambda} .\end{array}$ & $\begin{array}{l}\mu o / \varepsilon \cdot \pm 1 / \mu . \\
\mu \varepsilon / 0 \cdot \pm 1 / 1 .\end{array}$ & $\begin{array}{l}\mu\urcorner / q \cdot \pm 1 / 0 . \\
\mu \tau / \varepsilon \cdot \pm \cdot / q .\end{array}$ & يشيش مقآز خربى (درصد) \\
\hline $\begin{array}{l}*^{t} / 1 \cdot \pm m / 0 . \\
r V / 1 \cdot \pm m / 2 .\end{array}$ & $\begin{array}{l}r\urcorner / q . \pm r / \Lambda . \\
*_{r o / Q . \pm \varepsilon / 1 .}\end{array}$ & $\begin{array}{l}r \mathrm{r} / \Lambda \cdot \pm \varepsilon / V . \\
r \tau / \mu \cdot \pm \varepsilon / 0 .\end{array}$ & $\begin{array}{l}r V / \varepsilon \cdot \pm \varepsilon / 7 . \\
r V / r \cdot \pm \varepsilon / \mu .\end{array}$ & 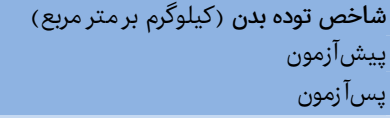 \\
\hline $\begin{array}{l}1 \cdot q / r \cdot \pm r q / \varepsilon . \\
1 \cdot 1 / \Lambda \cdot \pm r V / 0 .\end{array}$ & $\begin{array}{l}\Lambda 9 / \Lambda \cdot \pm \mu / / . \\
\Lambda / 0 . \pm r V / q .\end{array}$ & $\begin{array}{l}q \vee / \Lambda \cdot \pm r q / V . \\
\wedge q / r \cdot \pm r \cdot / \Lambda .\end{array}$ & $\begin{array}{l}1 \cdot 1 / \cdot \pm r r q / 9 . \\
1 \cdot . / 7 \cdot \pm r \wedge / \vee\end{array}$ & 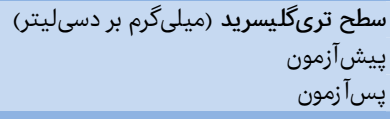 \\
\hline $\begin{array}{l}00 / 0 . \pm 11 / \varepsilon . \\
0 \wedge / 0 . \pm 9 / 9 .\end{array}$ & $\begin{array}{l}7 / / 7 \cdot \pm 1 \varepsilon / 7 . \\
7 \gamma / r \cdot \pm \mid \Lambda / \mu .\end{array}$ & $\begin{array}{l}0 V / T^{\mu} \cdot \pm 10 / 7 . \\
0 N / 1 \cdot \pm 1 \varepsilon / 9 .\end{array}$ & $\begin{array}{l}00 / V \cdot \pm 11 / T^{\mu} \\
0 \varepsilon / 7 \cdot \pm 1 \cdot 10\end{array}$ & 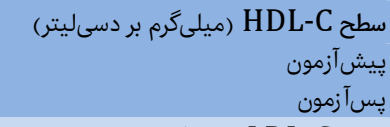 \\
\hline $\begin{array}{l}91 / V \cdot \pm 10 / V . \\
* V 7 / 1 \cdot \pm 1 V / 7 .\end{array}$ & $\begin{array}{l}\wedge V / 1 \cdot \pm I V / \varepsilon \\
*_{V Y / I \cdot \pm I V / \varepsilon}\end{array}$ & $\begin{array}{l}\Lambda \Lambda / r \cdot \pm 17 / 0 \\
\Lambda \cdot / \Lambda \cdot \pm 19 / \gamma\end{array}$ & $\begin{array}{l}\wedge V / \varepsilon \cdot \pm I V / r \cdot \\
\wedge 9 / 1 \varepsilon \pm r \mu / r^{\mu} .\end{array}$ & سيش سيش آزمون LDL-C \\
\hline $170 / \mu \cdot \pm r \cdot 10$. & $100 / r \cdot \pm 1 / / 1$ & $17 r / r \cdot \pm r \cdot / r \cdot$ & $10 N / \varepsilon \cdot \pm 1 V / 0$ & سيشطح كلسترول (ميلىگرم بر دسىليتر) \\
\hline$*_{10 \cdot / r \cdot \pm r r / 0 .}$ & 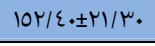 & $10 V / 1 \cdot \pm r / 9$. & $171 / 8 \cdot \pm 10 / 9$ & يسآزمون \\
\hline
\end{tabular}

p<•/.0*

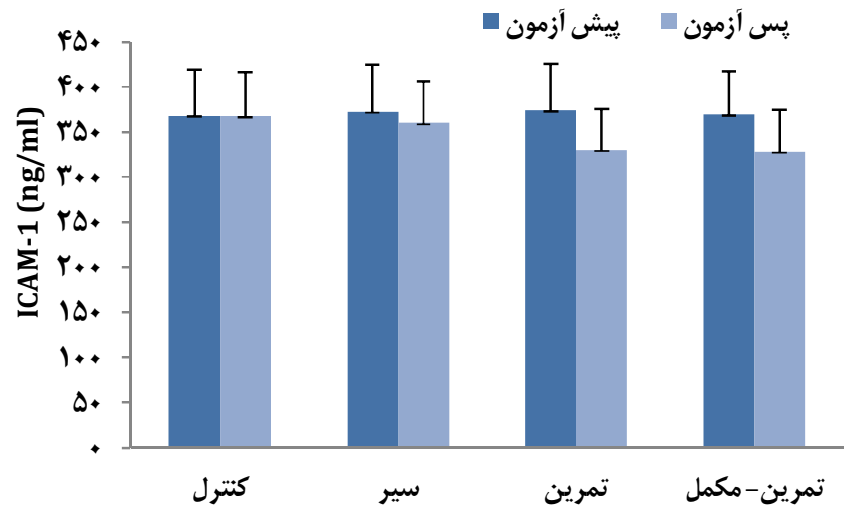

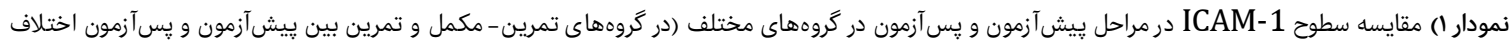

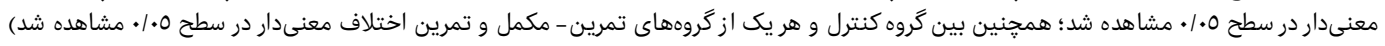

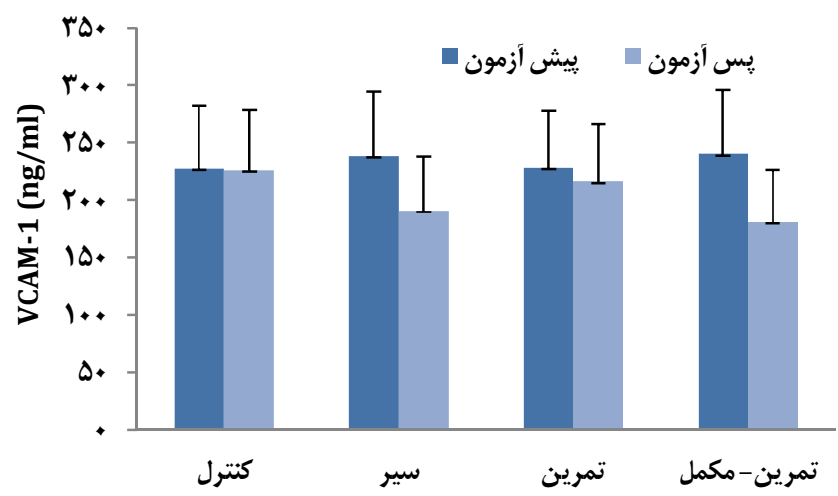

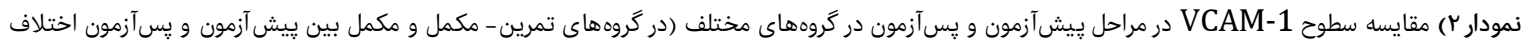

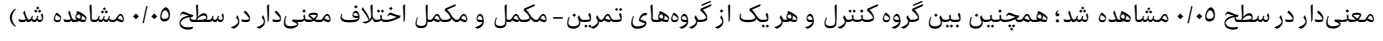




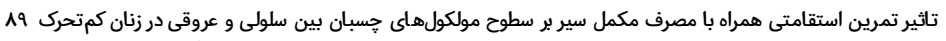

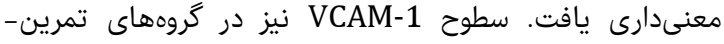

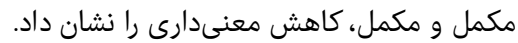

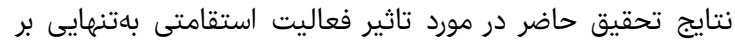

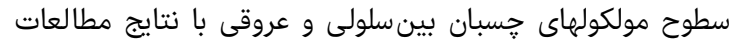

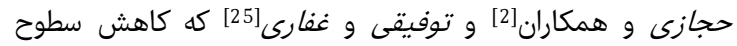
بدون كاهش معنىدار در سطوح مولكول ICAM-1

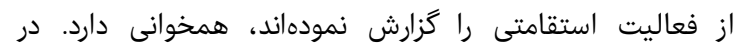

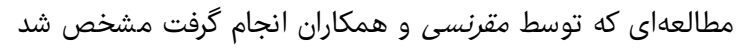

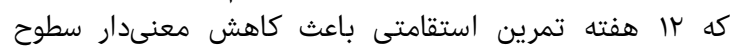

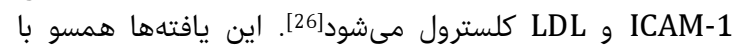

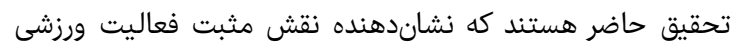

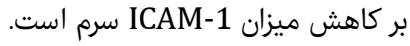

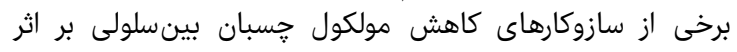

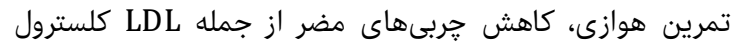

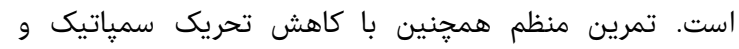

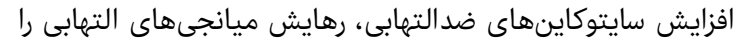

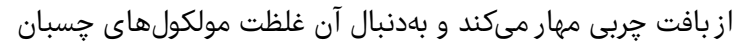

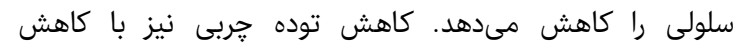

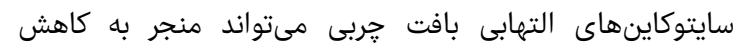

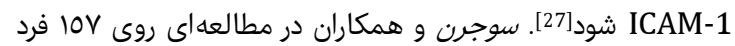

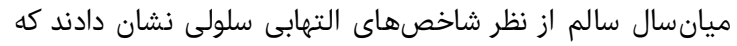

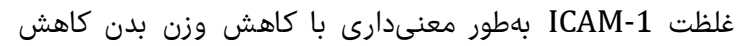

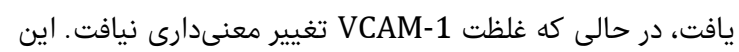
يزوهشكران كزارش كردند كه كاهش دال

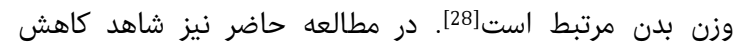

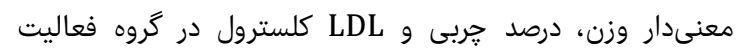

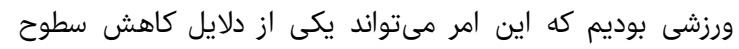
ICAM-1

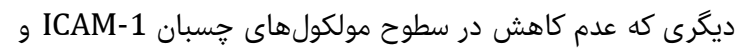

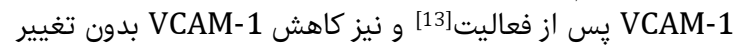
معنىدار در سطوح ICAM-1 را نشان دادهاند[14]، همخوانى ندارد.

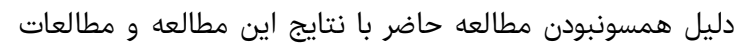

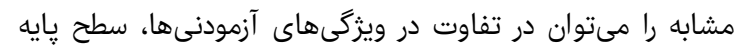

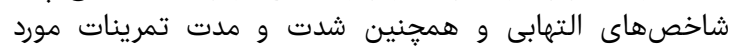

استفاده دانست.

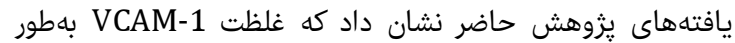

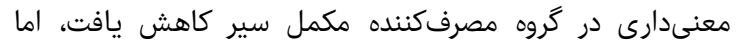

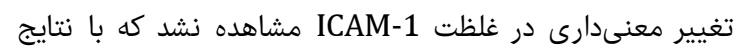

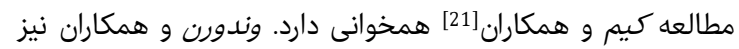

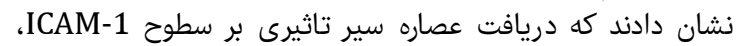
(يروتئين واكنشكر CRP

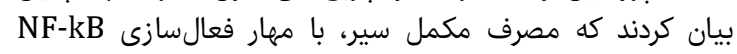

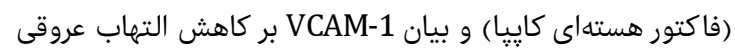

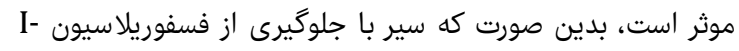

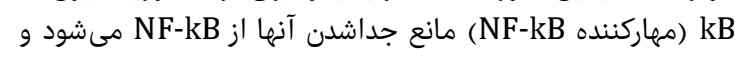

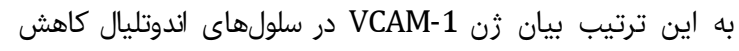

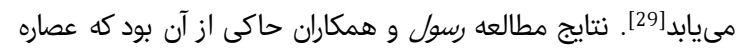

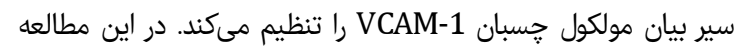

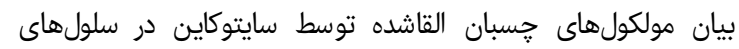

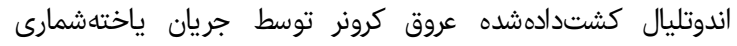
اندازهيرى شد. تكثير اين سلولها با عصاره سير بهطور معنى ادهارى بيان
يافتهها

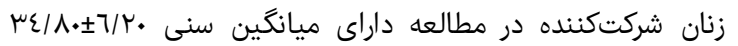

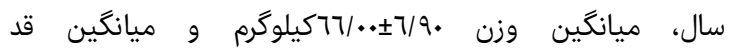
10V/1·×0/V.

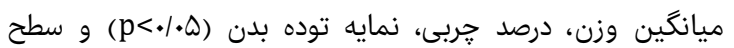

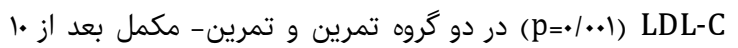

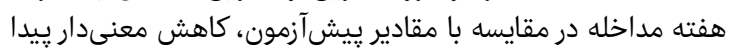

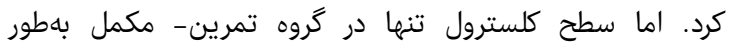

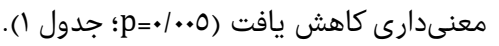

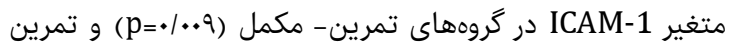

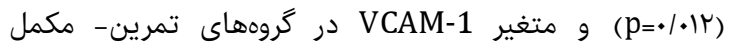

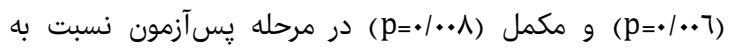

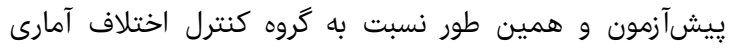

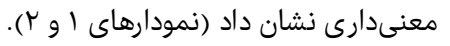

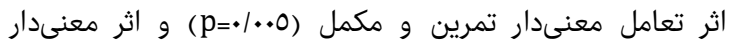

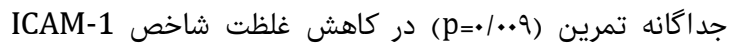

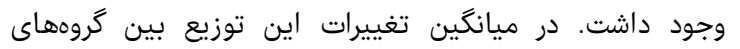

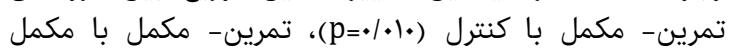

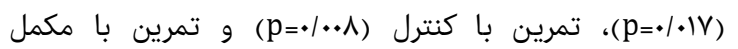
(p=•/•ll)

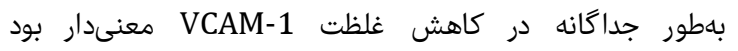

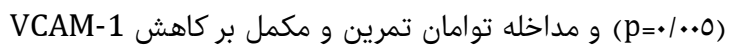

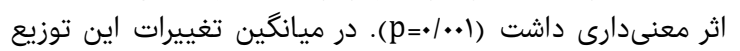

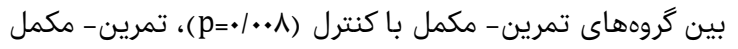

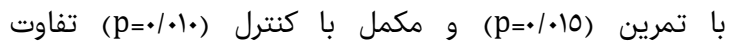
معنىدارى مشاهده شد.

بحث

مطالعات اخير نشان دادهاند كه ملكولهاى جسبان بين سلولى و

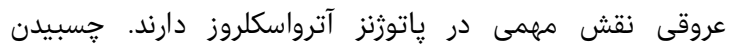

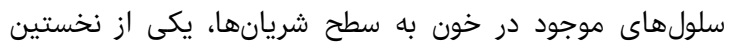

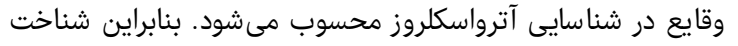

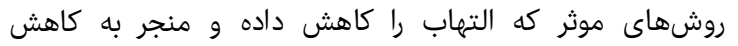

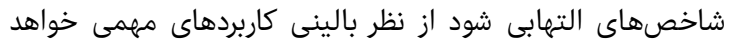

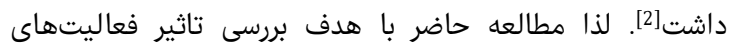

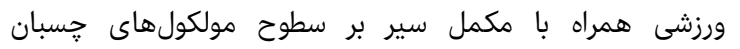

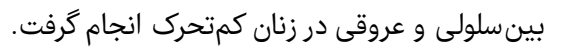

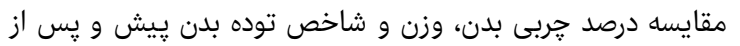

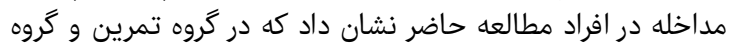

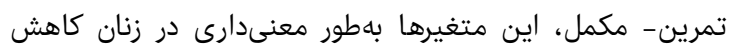

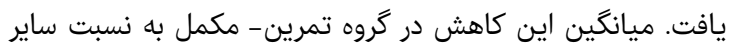

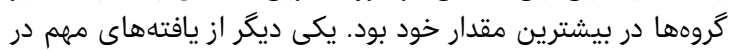

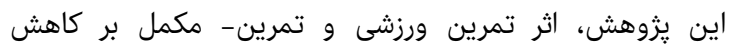

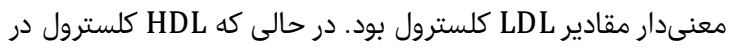

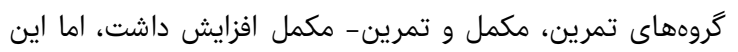

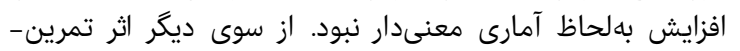

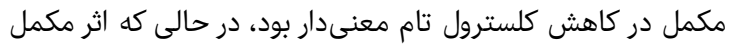

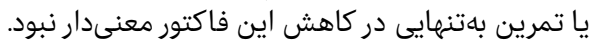

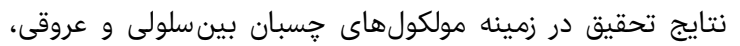

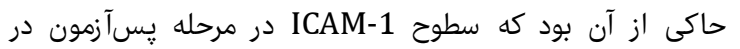

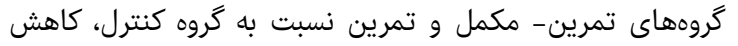




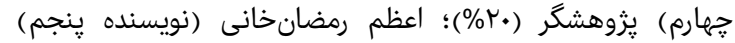

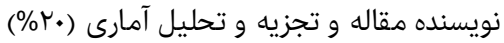

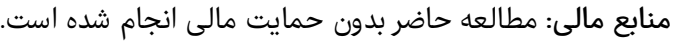

\section{منابع}

1- Bizheh N, Ebrahimi A, Jaafari M. The effects of three months aerobic exercise on novel atherosclerosis risk factors in untrained middle aged men. Glob J Sci Eng Technol. 2013;(5):158-70.

2- Hejazi SM, Abrishami LH, Khani JM, Boghrabadi V. The effects of 8-week aerobic exercises on serum levels of cell adhesion molecules among middle-aged women. Adv Stud Biol. 2013;5(6):279-89.

3- Bastien M, Poirier P, Lemieeux I, Després JP. Overview of epidemiology and contribution of obesity to cardiovascular disease. Prog Cardiovasc Dis. 2014;56(4):369-81.

4- Hammett CJ, Prapavessis H, Baldi JC, Varo N, Schoenbeck U, Ameratunga R, et al. Effects of exercise training on 5 inflammatory markers associated with cardiovascular risk. Am Heart J. 2006;151(2):367.e7-e16. 5- Kritchevsky SB, Cesari M, Pahor M. Inflammatory markers and cardiovascular health in older adults. Cardiovasc Res. 2005;66(2):265-75.

6- Golbidi S, Mesdaghinia A, Laher J. Exercise in the metabolic syndrome. Oxid Med Cell Longev. 2012;2012:349710.

7- Ding YH, Young CN, Luan X, Li J, Rafols JA, Clark JC, et al. Exercise preconditioning ameliorates inflammatory injury in ischemic rats during reperfusion. Acta Neuropathol. 2005;109(3):237-46.

8- Maeda S, Tanabe T, Otsuki T, Sugawara J, Lemitsu M, Miyauchi T, et al. Moderate regular exercise increases basal production of nitric oxide in elderly women. Hypertens Res. 2004;27(12):947-53.

9- Saxton JM, Zwierska K, Hopkinson E, Espigares S, Choksy S, Nawas S, et al. Effect of upper-lower- limb exercise training on circulation soluble adhertion molecules, hs-CRP and stress protein in pasint with cladication. Eur J Vasc Endovasc Surg. 2008;35(5):60713.

10- Linares-Segovia B, Guízar-Mendoza JM, AmadorLicona N, Barbosa-Sabanero G, Malacara JM. Effect of an exercise program, on hemodynamic, metabolic and inflammatory markers in obese Mexican adolescents. Endocrinol Metab Synd. 2013;S2:1-4.

11- Palmefors H, DuttaRoy S, Rundqvist B, Börjesson M. The effect of physical activity or exercise on key biomarkers in atherosclerosis--a systematic review. Atherosclerosis. 2014;235(1):150-61.

12- Moemen Kahkha H, Nasrabadi R, Nuraeinjar M. Effect of twelve weeks aerobic training on selected molecules TNF- $1 \alpha$, CRP, ICAM- 1 and VCAM- 1 in type 2 diabetes in middle-aged women. J Sci Res Dev. 2015;2(1):154-7.

13- Sabatier MJ, Schwark EH, Lewis R, Sloan G, Cannon J, McCully K. Femoral artery remodeling after aerobic exercise training without weight loss in women. Dyn Med. 2008;7:13.

14- Naji Esfahani $H$, Zamani M, Marandi SM, Shaygannejad V, Javanmard H. Preventive effects of a three-month yoga intervention on endothelial function in patients with migraine. Int J Prev Med. 2014;5(4):424-9.

15- Morihara N, Ushijima M, Kashimoto N, Sumioka I, Nishihama T, Hayama $M$, et al. Aged garlic extract ameliorates physical fatigue. Biol Pharm Bull. 2006;29(5):962-6.

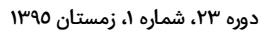

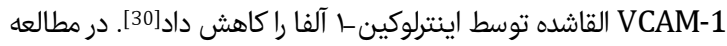
ديكرى نيز نشان داده شد كه عصاره سير سياه، بيان VCAM-1 توسط TNF-

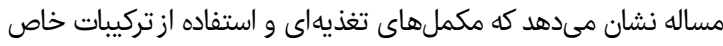

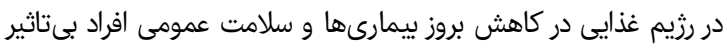
نيست] نئ.

نتايج مطالعه حاضر نشان داد كه غلظت ICAM-1 و

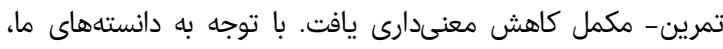

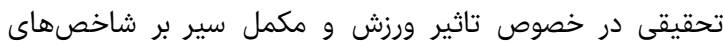

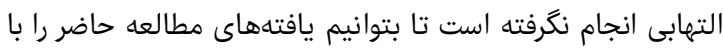

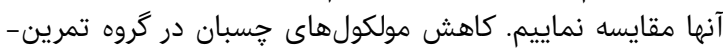

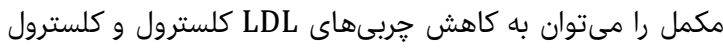

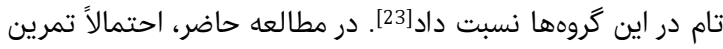

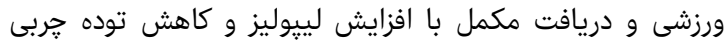

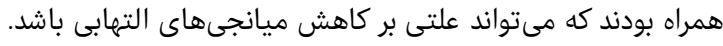

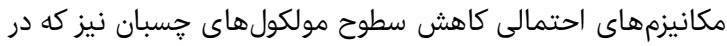

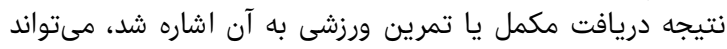

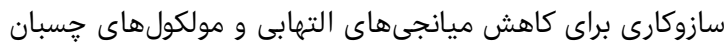

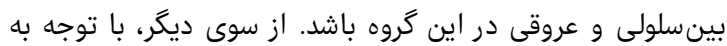

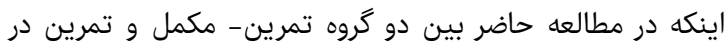

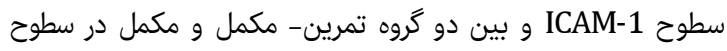

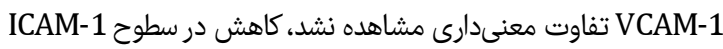

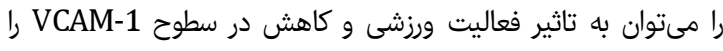

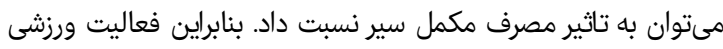
تاثير معنىدارى بر كاهش سطوح

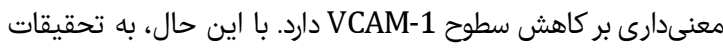

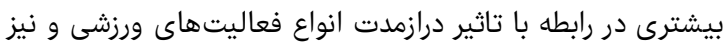

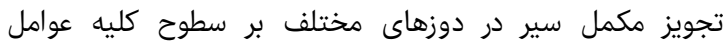

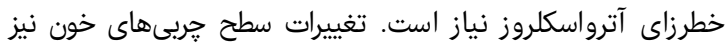

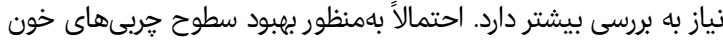

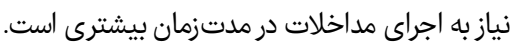

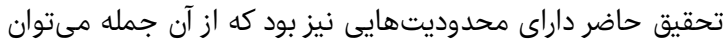

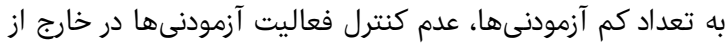

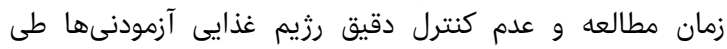

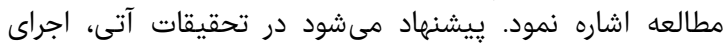

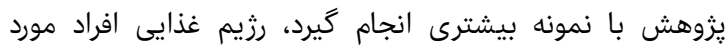

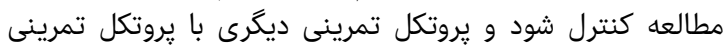
مطالعه حاضر مقايسه شود.

تثيجهكيرى •1 هفته فعاليت ورزشى همراه با مصرف مكمل سير، سطوح

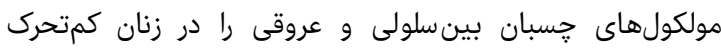
كاهش مىدهد.

تشكر و قدردانى: بدين وسيله از آزمودنىهاى ارجمند كه بدون

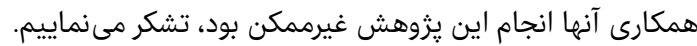

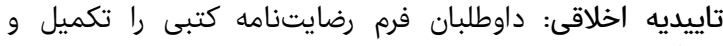

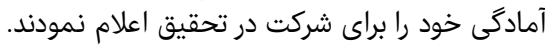

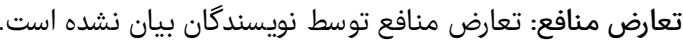

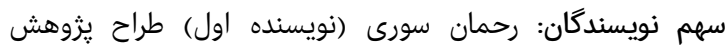

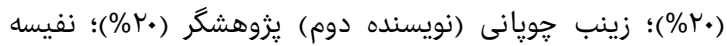

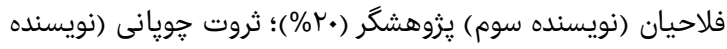
فصل نامه علمى - يُروهشى افق دانش 
91 تاثير تمرين استقامتى همراه با مصرف مكمل سير بر سطوح مولكولهاى حسبان بين سلولى و عروقى در زنان كمتحرك . ر .

garlic compounds. J Immunol Res. 2015;2015:13.

24- Choi KM, Kim TN, Yoo HJ, Lee KW, Cho GJ, Hwang TG,

et al. Effect of exercise training on A-FABP, lipocalin-2

and RBP4 levels in obese women. Clin Endocrinol. 2009;70(4):569-74.

25- Tofighi A, Ghafari G. Effect of Aerobic Training with Omega-3 consumption on soluble adhesion molecules in the obese women. Med J Tabriz Univ. 2013;36(1):20-7. [Persian]

26- Mogharnasi M, Gaeini AA, Sheikholeslami Vatani D. Effect of sprint training and detraining period on cellular adhesion molecule (sICAM-1) in wistar rats. Olympic. 2008;16(3):19-30. [Persian]

27- Monchanin G, Serpero LD, Connes P, Tripette J, Woassi D, Bezin L, et al. Effects of progressive and maximal exercise on plasma levels of adhesion molecules in athletes with sickle cell trait with or without alphathalassemia. J Appl Physiol. 2007;102(1):169-73.

28- Sjögren P, Cederholm T, Heimbürger M, Stenvinkel P, Vedin I, Palmblad J, et al. Simple advice on lifestyle habits and long-term changes in biomarkers of inflammation and vascular adhesion in healthy middle-aged men. Eur J Clin Nutr. 2010;64(12):1450-6.

29- van Doorn MB, Espirito Santo SM, Meijer $P$, Kamerling IM, Schoemaker RC, Dirsch V, et al. Effect of garlic powder on C-reactive protein and plasma lipids in overweight and smoking subjects. Am J Clin Nutr. 2006;84(6):1324-9.

30- Rassoul F, Salvetter J, Reissig D, Schneider W, Thiery J, Richter V. The influence of garlic (Allium sativum) extract on interleukin 1alpha-induced expression of endothelial intercellular adhesion molecule-1 and vascular cell adhesion molecule-1. Phytomedicine. 2006;13(4):230-5.

16- Ebrahim Kh, Ahmadizad S, Ghanimati R, Bagheri A Sheikhi S, Ghanimati M. The effect of endurance training and garlic consumption on vo2max in non-active men. Manag Appl Res Biol Sci Sport. 2011;1(2):11-8. [Persian] 17- Lee YM, Gweon OC, Seo YJ, Im J, Kang MJ, Kim MJ, et al. Antioxidant effect of garlic and aged black garlic in animal model of type 2 diabetes mellitus. Nutr Res Pract. 2009;3(2):156-61.

18- Morris DM, Beloni RK, Wheeler HE. Effects of garlic consumption on physiological variables and performance during exercise in hypoxia. Appl Physiol Nutr Metab. 2013;38(4):363-7.

19- Saki B, Paydar Ardakani SM, Amraei Z, Salehi Abarghuei A. The effect of garlic supplementation on aerobic performance in non-athlete men. Iran J Nutr Sci Food Technol. 2015;10(2):115-20.

20- Vazquez-Prieto MA, Rodriguez Lanzi C, Lembo C, Galmarini CR, Miatello RM. Garlic and onion attenuates vascular inflammation and oxidative stress in fructosefed rats. J Nutr Metab. 2011;2011:7.

21- Kim SR, Jung YR, An HJ, Kim DH, Jang EJ, Choi YJ, et al. Anti-wrinkle and anti-inflammatory effects of active garlic components and the inhibition of MMPs via NF- $\kappa B$ signaling. PLoS One. 2013;8(9):e73877.

22- Lee EN, Choi YW, Kim HK, Park JK, Kim HJ, Kim MJ, et al. Chloroform extract of aged black garlic attenuates TNF- $\alpha$-induced ROS generation, VCAM-1 expression, NF$\kappa \mathrm{B}$ activation and adhesiveness for monocytes in human umbilical vein endothelial cells. Phytother Res. 2011;25(1):92-100.

23- Arreola R, Quintero-Fabián S, López-Roa RI, FloresGutiérrez EO, Reyes-Grajeda JP, Carrera-Quintanar L, et al. Immunomodulation and anti-inflammatory effects of 